\title{
Central Modulatory Neurons Control Fuel Selection in Flight Muscle of Migratory Locust
}

\author{
Tim Mentel, ${ }^{1 \star}$ Carsten Duch, ${ }^{1 *}$ Heike Stypa,${ }^{2}$ Gerhard Wegener, ${ }^{2}$ Uli Müller, ${ }^{1}$ and Hans-Joachim Pflüger ${ }^{1}$ \\ ${ }^{1}$ Institute of Biology/Neurobiology, Free University Berlin, 14195 Berlin, Germany, and ${ }^{2}$ Institute of Zoology, Department of Molecular Physiology, \\ Johannes-Gutenberg University, 55099 Mainz, Germany
}

\begin{abstract}
Insect flight is one of the most intense and energy-demanding physiological activities. High carbohydrate oxidation rates are necessary for take-off, but, to spare the limited carbohydrate reserves, long-distance flyers, such as locusts, soon switch to lipid as the main fuel. We demonstrate that before a flight, locust muscles are metabolically poised for take-off by the release of octopamine from central modulatory dorsal unpaired median (DUM) neurons, which increases the levels of the potent glycolytic activator fructose 2,6-bisphosphate in flight muscle. Because DUM neurons innervating the flight muscles are active during rest but selectively inhibited during flight, they stimulate carbohydrate catabolism during take-off but tend to decrease muscle glycolysis during prolonged flight. cAMP-dependent protein kinase A is necessary but not sufficient for signal transduction, suggesting parallel control via a calcium-dependent pathway. Locust flight is the first reported instance of a direct and specific involvement of neuronal activity in the control of muscle glycolysis in working muscle during exercise.
\end{abstract}

Key words: insect; octopamine; metabolism; motor behavior; glycolysis; neuromuscular system; modulation

\section{Introduction}

In flying locusts, the ATP-turnover rate in flight muscles is increased $\sim 100$-fold compared with rest. Carbohydrate is a major fuel early in flight, but during prolonged flight, the muscles soon switch to oxidizing lipids to spare limited carbohydrate stores (Wegener, 1996; Candy et al., 1997). Locusts are formidable longdistance flyers, as demonstrated by monitoring swarms with satellites (Krall et al., 1997). Prolonged flight requires two main metabolic adjustments. First, lipids must be made available. Lipid mobilization is induced by increasing hemolymph levels of adipokinetic hormones (Van der Horst, 1990; Van der Horst et al., 2001). Second, catabolism in working flight muscles must shift from carbohydrate to fat oxidation. This requires a yet unknown specific mechanism to decrease glycolytic flux, because locusts still have considerable carbohydrate stores after $30 \mathrm{~min}$ of flight, when carbohydrate oxidation has dropped to $<10 \%$ of the initial rate (Jutsum and Goldsworthy, 1976). The main control element of glycolysis is phosphofructokinase (PFK), which is inhibited by physiological concentrations of ATP and activated by AMP (Wegener et al., 1987). However, the levels of these effectors in muscle change only during the first $2 \mathrm{sec}$ after take-off but remain constant thereafter, as demonstrated by ${ }^{31} \mathrm{P}$ nuclear magnetic resonance (NMR) spectroscopy (Wegener et al., 1991). Therefore, they do not cause the decreased PFK activity during prolonged

\footnotetext{
Received 0ct. 17, 2002; revised Nov. 22, 2002; accepted Nov. 24, 2002.

This study was supported by Deutsche Forschungsgemeinschaft Grants Pf 128/13-1 (T.M.), DU331/2-2 (C.D.), We 494/10-2 (G.W.), SFB 515,C3 (U.M.), and SFB 515, B6 (H.-J.P.). We thank Dr. R. B. Levine (ARL Division of Neurobiology, University of Arizona, Tucson, AZ) for helpful suggestions on this manuscript.

T.M. and C.D. contributed equally to this work.

Correspondence should be addressed to Carsten Duch, Institute of Biology/Neurobiology, Free University Berlin, Koenigin-Luise Strasse 28-30, 14195 Berlin, Germany. E-mail: duch@neurobiologie.fu-berlin.de. Copyright $\odot 2003$ Society for Neuroscience $\quad 0270-6474 / 03 / 231109-05 \$ 15.00 / 0$
}

flight. In contrast, the levels of another potent activator of PFK, fructose 2,6-bisphosphate $\left(\mathrm{F} 2,6 \mathrm{P}_{2}\right)$, decrease by $80 \%$ within 10 min of flight (Wegener et al., 1986). F2,6 $\mathrm{P}_{2}$ acts synergistically with AMP in activating PFK and is neither a substrate nor an intermediate of any metabolic pathway, but rather an intracellular messenger responding to extracellular signals. The important question, how $\mathrm{F} 2,6 \mathrm{P}_{2}$ is controlled during exercise, remains unanswered for vertebrate and insect muscle (Wegener, 1996; Krause and Wegener, 1996; Wegener and Krause, 2002).

In isolated perfused locust flight muscle, $\mathrm{F} 2,6 \mathrm{P}_{2}$ levels are increased within 5 min by octopamine (Blau et al., 1994), and injection of high doses of octopamine neutralizes flight-induced decrease in F2,6 $\mathrm{P}_{2}$ (Blau and Wegener, 1994). After 10 min of flight, octopamine levels in flight muscles are decreased, whereas hemolymph octopamine is increased (Goosey and Candy, 1980, 1982; Orchard et al., 1993). Therefore, octopamine and F2,6 $\mathrm{P}_{2}$ in flight muscle are correlated also in vivo, and octopamine levels in working flight muscle are controlled independently of hemolymph octopamine. The only source for octopamine release onto flight muscles is central dorsal unpaired median (DUM) modulatory neurons with bilaterally symmetrical projections on both sides of the body. DUM neuron terminals are surrounded by tight glial sheaths, suggesting precise release onto muscles and effective compartmentalization between muscle and hemolymph octopamine (Rheuben, 1995). DUM neurons innervating wing muscles are active in resting locusts but are inhibited during flight (Duch and Pflüger, 1999). We show that DUM neuron activity increases $\mathrm{F} 2,6 \mathrm{P}_{2}$ levels in resting flight muscles, thus preparing them for high glycolytic rates, as required for take-off. Conversely, inhibition of DUM neurons during flight corresponds to decreased muscle octopamine levels, most likely contributing to decreased glycolysis during prolonged flight. 


\section{Materials and Methods}

Animals. Adult locusts (Locusta migratoria) of either sex were taken from a crowded laboratory colony at the Free University of Berlin.

Stimulation of DUM neurons innervating flight muscles. Because of their bilaterally symmetrical projection patterns, DUM neurons innervate all six major flight muscles on both sides of the thorax. Because no other efferent thoracic neuron shows bilaterally symmetrical projections, DUM neurons can be stimulated selectively, thus inducing octopamine release onto specific flight muscles without stimulating the motoneurons innervating these muscles. To test whether octopamine release from DUM neurons affected flight muscle metabolism, DUM neurons were stimulated antidromically, and the content of $\mathrm{F} 2,6 \mathrm{P}_{2}$ was measured in one of the three wing elevator muscles, M119, according to Albrecht (1953). Stimulations were conducted in restrained semi-intact preparations. Animals were fixed in plasticine dorsal side up, and the thorax was dissected open along the dorsal midline. The gut was removed, and the electrodes for extracellular recording and stimulation were placed at selected nerves. To induce octopamine release on M119 on one side only, nerve 4 was cut on one side, and the four DUM neurons innervating M119 were stimulated antidromically via their axons with a suction electrode (see Fig. 1A, stimulation electrode 1). This induced octopamine release onto muscle M119 on the contralateral side to the stimulation electrode. The denervated M119 on the ipsilateral side to the stimulation electrode served as internal control (see Fig. $1 A$ ). Individual pulses of 3 $\mathrm{nA}$ amplitude and $0.1 \mathrm{msec}$ duration were applied every second for 20 min. Successful stimulation was monitored by recording the DUM neuron spikes extracellularly with a hook electrode from the contralateral uncut nerve 4 (see Fig. $1 A$ ).

To induce release of octopamine onto M119 on both sides of the thorax without affecting the motoneurons to M119, DUM neurons were stimulated antidromically via nerve 3 with a suction electrode (see Fig. $1 A$, stimulation electrode 2). Antidromic stimulation of nerve 3 elicited spikes in all four DUM neurons innervating M119, because they all possess axonal projections in nerve 3 and in nerve 4 (Kutsch and Schneider, 1987).

After stimulation, the muscles M119 were removed from both sides (experimental and control muscle). For enzymatic detection of the content of $\mathrm{F} 2,6 \mathrm{P}_{2}$, muscles were frozen in liquid nitrogen within 15-20 sec after electrical stimulation and subsequently treated as described below. For in vitro phosphorylation assays, muscles were frozen onto the tip of a steel pestle that had been cooled in liquid nitrogen. Then the pestle with the frozen muscle on its tip was placed into a microcapillary of $1 \mathrm{~mm}$ inner diameter (100 $\mu$ l disposable micropipettes; Blaubrand) that contained $20 \mu \mathrm{l}$ of frozen Tris-HCl buffer ( $50 \mathrm{~mm}$ ), pH 7.5, at the opposite end. The muscle was crushed inside the microcapillary without thawing by tipping the steel pestle on the surface of the frozen buffer. After crushing, microcapillaries were stored in liquid nitrogen until phosphorylation assays were conducted as described below.

Assay of F2, $6 \mathrm{P}_{2}$. To prevent the degradation of $\mathrm{F} 2,6 \mathrm{P}_{2}$ (which is very labile at low $\mathrm{pH}$ but stabile in alkali), frozen flight muscles were swiftly homogenized in 10 parts $(\mathrm{v} / \mathrm{w})$ of $20 \mathrm{~mm} \mathrm{NaOH}$ by sonication. The homogenate was incubated at $80^{\circ} \mathrm{C}$ for $5 \mathrm{~min}$, and then centrifuged at $10,000 \times g$ for $15 \mathrm{~min}$. F2, $6 \mathrm{P}_{2}$ was assayed in the supernatant on the basis of its ability to activate the pyrophosphate-dependent PFK ( $\left.\mathrm{PP}_{\mathrm{i}}-\mathrm{PFK}\right)$, which we had purified from potato tubers according to Van Schaftingen et al. (1982). $\mathrm{PP}_{\mathrm{i}}-\mathrm{PFK}$ is strongly activated by $\mathrm{F} 2,6 \mathrm{P}_{2}$, whereas other activators of animal PFKs have virtually no effect on the enzyme. A

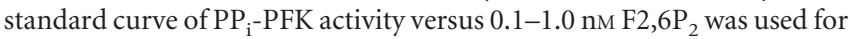
the readings (Van Schaftingen, 1984).

In vitro phosphorylation assay. PKA activity in flight muscle M119 (see Fig. $1 A$ ) was measured by a fast in vitro phosphorylation assay using phosphatase inhibitor 1 (I-1) as a PKA substrate (Hildebrandt and Müller, 1995). In initial experiments, we confirmed that I-1 purified from bovine brain is a specific substrate for cAMP-dependent protein kinase of locust muscle. For the phosphorylation assay, samples $(10 \mu \mathrm{l})$ in the microcapillaries were thawed and, just before complete melting, plunged into the phosphorylation mixture $(10 \mu \mathrm{l})$, which comprised $1 \mu \mathrm{Ci}$ $\left[\gamma^{-32} \mathrm{P}\right]$ ATP $(5000 \mathrm{Ci} / \mathrm{mmol}), 20 \mu \mathrm{M}$ ATP, 1 mm EGTA, 10 mM mercap- toethanol in $50 \mathrm{~mm}$ Tris- $\mathrm{HCl}, \mathrm{pH} 7.5$, and an aliquot of the heat stable I-1 $(1 \mu \mathrm{g})$, boiled for $2 \mathrm{~min}$ before use. After incubation for $80 \mathrm{sec}$ at room temperature $\left(20^{\circ} \mathrm{C}\right)$, reactions were stopped by adding $6 \mu \mathrm{l}$ of sample buffer $(0.25$ м Tris-HCl, pH 6.8, containing 5\% mercaptoethanol, 5\% SDS, $20 \%$ glycerol, and $0.1 \%$ bromphenol blue). SDS-PAGE and autoradiography were performed as described by Hildebrandt and Müller (1995). Autoradiographs were scanned, and the density of both the PKAspecific I-1 band and the bands of the intrinsic proteins were determined using NIH Image. In each sample, the ${ }^{32} \mathrm{P}$ incorporation into the PKAspecific substrate I-1 was normalized with respect to the total ${ }^{32} \mathrm{P}$ incorporation into intrinsic proteins (because of other kinase activities) [see Fig. $2 \mathrm{~A}$, black arrow (for ${ }^{32} \mathrm{P}$ incorporation into PKA-specific substrate), white arrowhead (for ${ }^{32} \mathrm{P}$ incorporation into intrinsic proteins)].

Injections into muscle M119. In some experiments, chemicals were injected into flight muscle M119 with a Hamilton syringe $(20 \mu \mathrm{l}$ of a $10^{-3}$ M solution in DMSO of the PKA inhibitor KT5720 or 8-bromocAMP; Calbiochem, San Diego, CA). Contralateral control muscles were injected with similar volumes of DMSO (Sigma, St. Louis, MO). Flight muscles are compact, with thickly packaged muscle bundles. If a dye is injected into such a muscle, it quickly spreads within the muscle but does not easily travel to adjacent muscles.

\section{Results}

Activity of DUM neurons increases the levels of F2,6 $P_{2}$ in flight muscle

DUM neurons are modulatory neurons intimately involved in insect flight (Orchard et al., 1993; Duch and Pflüger, 1999). They

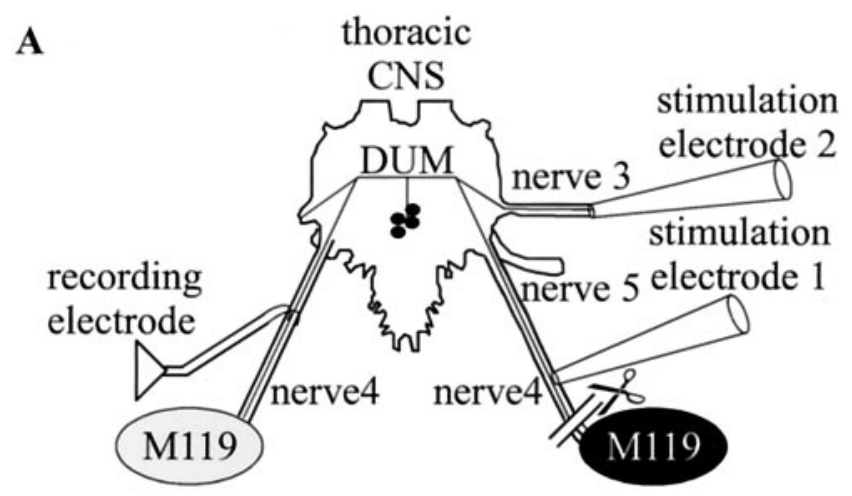

B

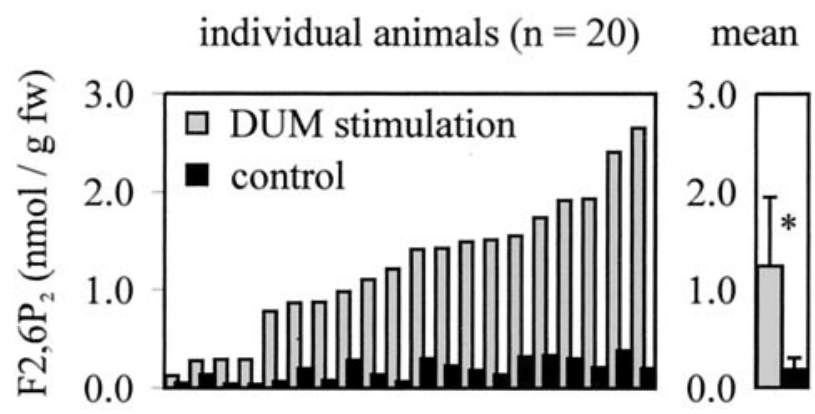

Figure 1. Effects of DUM neuron activity on the glycolytic activator $\mathrm{F} 2,6 \mathrm{P}_{2}$ in flight muscle. $A$, Schematic drawing of the stimulation protocol. The four cell bodies of the DUM neurons to the flight muscles M119 are represented by black circles; their axons are represented by straight lines. After denervating the right flight muscle M119, stimulation via electrode 1 induces octopamine release from DUM neurons onto the left M119 only (see Results and Figs. $1 B, 2 A, B$ ). Stimulation success is recorded on the left nerve 4 . Stimulating nerve 3 without cutting nerve 4 induces octopamine release on the muscles M119 on both sides (see Results, Figs. 2C, 3). B, The content of $F 2,6 P_{2}$ in nanomoles per gram of fresh weight $(g f w)$ in flight muscle $M 119$ is increased by DUM neuron activity (gray bars; stimulation via nerve 4 at $1 \mathrm{~Hz}$ for $20 \mathrm{~min}$ ) compared with the contralateral control muscle from the same locust (black bars). Means + SDs are shown on the right. ( $t$ test; $n=29 ;{ }^{*} p<0.001$ ). 


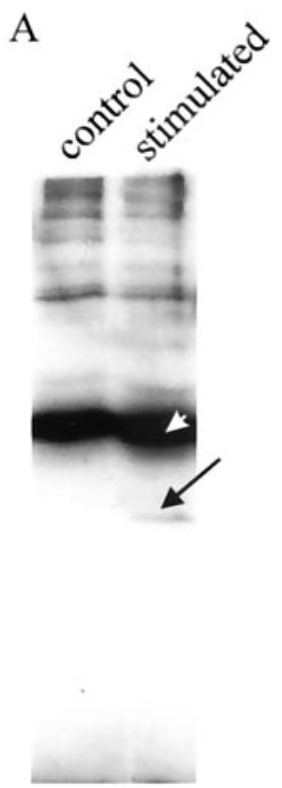

B

$$
\begin{aligned}
& \text { A1: DUM + }(+) \\
& \text { A2: DUM - }(-) \\
& \text { B1: DUM -, bromo-cAMP injection }(-, \text { cA) } \\
& \text { B2: DUM -, control injection }(-, \mathrm{c}) \\
& \text { C1: DUM +, control injection }(+, \mathrm{c}) \\
& \text { C2: DUM +, KT5720 injection }(+, \mathrm{K})
\end{aligned}
$$

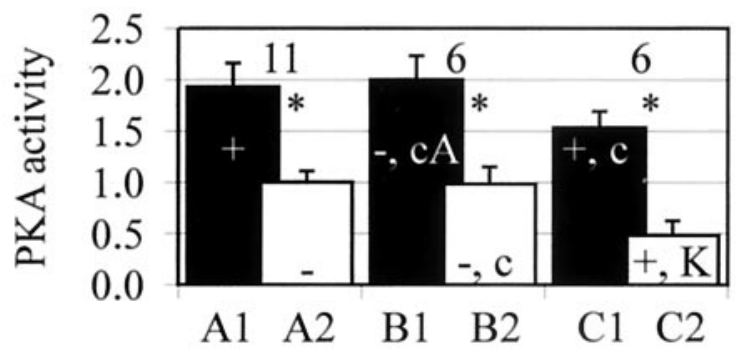

Figure 2. DUM neuron activity increases PKA activity in flight muscles. A, Phosphorylation assay for PKA activity in flight muscle M119 on the control (left) and on the experimental side (right). The band for ${ }^{32}$ P incorporation into the PKA-specific substrate I-1 is marked with a black arrow; the band for ${ }^{32} \mathrm{P}$ incorporation into intrinsic proteins is marked with a white arrowhead. $B$, Bar diagram showing the effects of DUM neuron activity ( $A 1$, muscle M119 affected by DUM neuron activity; $A 2$, control) and of an injected cAMP donor (B1, injected 8-bromo-CAMP; B2, sham injected with DMSO) on PKA activity in flight muscle M119. The increase in PKA activity mediated by DUM neuron activity $(C 1$, muscle M119 affected by DUM neuron activity and injected with DMSO) is abolished by injections of the PKA inhibitor KT5720 ( $(2$, muscle M119 affected by DUM neuron activity after injection of KT5720). Means + SDs are given, with asterisks indicating significant differences. ( $t$ test; $p<0.01)$.

supply the flight muscles and affect contraction kinetics but do not trigger contraction. We tested whether activity of DUM neurons supplying flight muscles also affected muscle metabolism and fuel selection. After unilateral denervation, DUM neurons were stimulated antidromically at $1 \mathrm{~Hz}$ for $20 \mathrm{~min}$ to induce octopamine release on the wing elevator muscle M119 on one side only (Fig. 1A). The contralateral M119 served as internal control (Fig. $1 A$, stimulation electrode 1). The levels of $\mathrm{F} 2,6 \mathrm{P}_{2}$ in flight muscle were increased by the activity of DUM neurons, demonstrating for the first time a direct and specific involvement of central modulatory neurons in the control of glycolysis and hence fuel selection in muscle during exercise. The stimulation frequency of $1 \mathrm{~Hz}$ is at the upper range of the firing frequencies recorded from semi-intact locust preparations during rest (Duch and Pflüger, 1999). The content of F2,6 $\mathrm{P}_{2}$ in muscles showed individual variation, both in the stimulated and unstimulated flight muscles. However, in all cases stimulation markedly increased the content of F2,6 $\mathrm{P}_{2}$ in flight muscle compared with the unstimulated control muscle (Fig. $1 B$ ). Thus DUM neuron activity produced a highly significant increase in the content of F2,6 $\mathrm{P}_{2}$, which was sevenfold on average (paired Student's $t$ test; $p<0.001$ ). This showed that DUM neuron activity in preflight locusts would be sufficient to maintain high levels of $\mathrm{F} 2,6 \mathrm{P}_{2}$, thus keeping the flight muscles poised for high glycolytic activity and hence ready for starting a flight.

In contrast, the previously measured decreases in octopamine and $\mathrm{F} 2,6 \mathrm{P}_{2}$ in active flight muscles (Goosey and Candy, 1982; Wegener et al., 1986) correspond to the inhibition of DUM neurons during flight (Duch and Pflüger, 1999). Because all other effectors of PFK are unchanged during flight, decreases in $\mathrm{F} 2,6 \mathrm{P}_{2}$ will attenuate glycolytic flux, as has been shown in an enzyme assay with PFK purified from locust flight muscle (Wegener et al., 1986, 1987).
Activity of DUM neurons increases PKA activity in flight muscle

We also tested whether the metabolic effect of DUM neuron activity was mediated by the same second messengers that mediate presynaptic and postsynaptic modulatory effects of octopamine on muscle contraction kinetics (Evans and Robb, 1993). At least two types of octopamine receptors have been described on locust skeletal muscles. They either increase intracellular calcium via the $\mathrm{IP}_{3}$ pathway or increase cAMP through adenylate cyclase activity, the latter activating PKA (Evans and Robb, 1993). With the same stimulation patterns as before, we followed PKA activity with substrate phosphorylation assays (Fig. 2A). DUM neuron activity significantly increased PKA activity in the flight muscle M119 (Fig. 2A,B). Injections of the CAMP donor bromo-8-cAMP into nonstimulated muscles had an effect on PKA activity that was similar to that seen for stimulations of DUM neurons (Fig. 2B). Stimulating DUM neurons via nerve 3 induced octopamine release on M119 on both sides of the body (see Materials and Methods) (Fig. 1A). In such preparations, injections of the PKA inhibitor KT5720 into one of the two M119 flight muscles decreased PKA activity in that muscle significantly (Fig. $2 B, C$ ) (Student's $t$ test; $p<0.001$ ) and thus abolished the effect of DUM neuron stimulation on PKA activity (Fig. $2 B, C$ ).

\section{PKA is necessary but not sufficient for signal transduction} from octopamine to $\mathrm{F} 2,6 \mathrm{P}_{2}$

We also tested whether the PKA pathway was necessary to mediate the effect of DUM neuron activity on F2,6 $\mathrm{P}_{2}$ content in flight muscle. DUM neurons were stimulated via nerve 3 (Fig. $1 \mathrm{~A}$, stimulation 2), with no nerves cut, so that octopamine was released on the muscles M119 on both sides. Before stimulation, one M119 was injected with the PKA blocker KT5720; the contralateral one was injected with DMSO (control). After stimulation, the muscles injected with KT5720 contained significantly less $\mathrm{F} 2,6 \mathrm{P}_{2}(p<0.01)$ than the control muscles (Fig. 3A), demonstrating that the CAMP-PKA pathway was necessary for signal transduction. To test whether increased PKA activity was sufficient to increase $\mathrm{F} 2,6 \mathrm{P}_{2}$ in muscle without DUM neuron stimulation, the cAMP donor bromo-8-cAMP was injected in M119 on one side only. The content of F2,6 $\mathrm{P}_{2}$ was measured in both muscles M119 from the same locusts after $20 \mathrm{~min}$. Although PKA activity was thus increased pharmacologically to a similar degree as by DUM neuron stimulation (Fig. $2 B$ ), this was not sufficient to increase $\mathrm{F} 2,6 \mathrm{P}_{2}$ in muscle M119 (Fig. $3 B$ ), indicating that a parallel pathway was involved in signal transduction from octopamine to $\mathrm{F} 2,6 \mathrm{P}_{2}$. Given the octopamine receptor complement of locust flight muscles (Evans and Robb, 1993), this is likely to be the $\mathrm{IP}_{3}$-calcium-dependent pathway. Whether both pathways are coupled to different receptor subtypes or whether the same octopamine receptor is coupled to different second messenger systems remains an open question (Robb et al., 1994). 
A

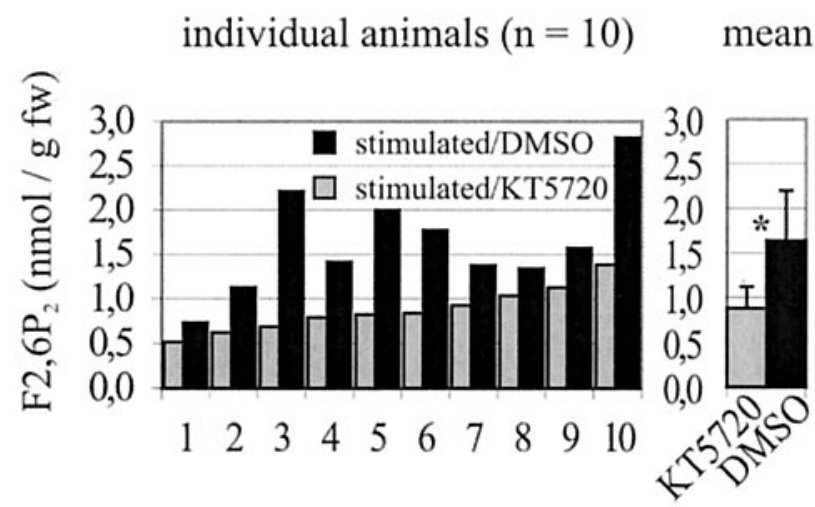

B

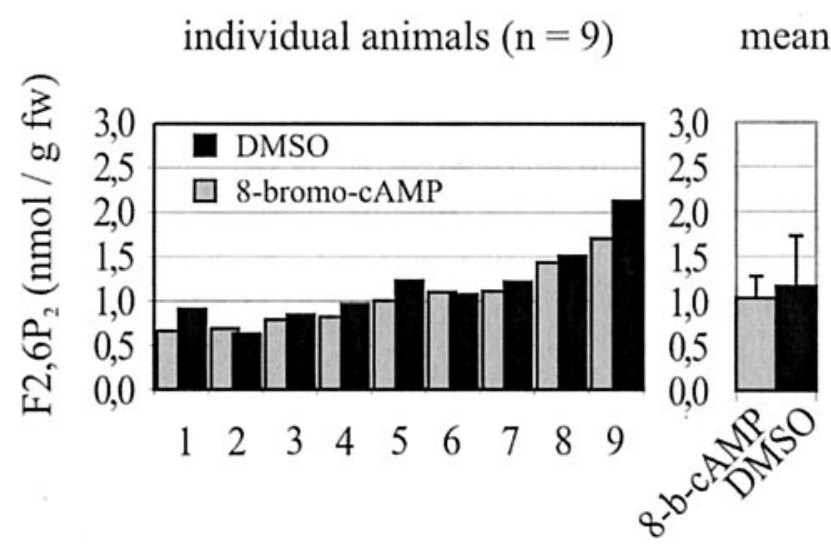

Figure 3. The CAMP-PKA pathway is necessary but not sufficient for DUM neuron activitymediated increases in $\mathrm{F} 2,6 \mathrm{P}_{2}$. A, DUM neurons were stimulated antidromically for 20 min with $1 \mathrm{~Hz}$ so that octopamine was released on M119 on both sides of the thorax. Injection of the PKA inhibitor KT5720 attenuated the effect of DUM neuron activity to increase the levels of $\mathrm{F}_{2}, 6 \mathrm{P}_{2}$ in nanomoles per gram of fresh weight ( $g f w$ ) in muscle M119 (white bars) compared with control injections with DMSO (black bars). B, Injection of the CAMP donor 8-bromo-CAMP (white bars) into muscles that were not affected by DUM neuron activity did not increase the content of $\mathrm{F} 2,6 \mathrm{P}_{2}$ compared with control injected muscles (black bars). Means $+\mathrm{SD}$ s of experiments in $A$ and $B$ are shown in bar diagrams on the right $\left(t\right.$ test; $\left.{ }^{*} p<0.01\right)$.

\section{Discussion}

It has long been known that muscle contraction triggered by the activity of motoneurons stimulates muscle catabolism. The balance of hydrolysis and resynthesis of ATP is controlled by intracellular feedback mechanisms in which ATP and its degradation products are involved (for review, see Wegener, 1996). In contrast, little is known as to the role of the nervous system in muscle metabolism during exercise.

Our results show that locust muscle catabolism can be affected by the activity of central modulatory neurons, which in turn are directly connected to the central neural circuits that produce flight motor output (Duch and Pflüger, 1999). In semi-intact preparations of locusts, DUM neurons that innervate flight muscles fire with frequencies between 0.1 and $1 \mathrm{~Hz}$ during rest (Duch and Pflüger, 1999). This study shows that a firing rate of $1 \mathrm{~Hz}$ is sufficient to maintain high levels of $\mathrm{F} 2,6 \mathrm{P}_{2}$ in flight muscle. However, in resting locusts this will not trigger glycolytic activity, because $\mathrm{F} 2,6 \mathrm{P}_{2}$ acts synergistically with AMP in activating PFK (Wegener et al., 1987), and the AMP content is very low in resting muscle (Wegener et al., 1991). As a consequence, preflight DUM neuron activity keeps the flight muscles poised for high glycolytic activity as required for take-off without wasting carbohydrate. With the onset of a flight, however, ATP turnover is increased dramatically, and this will cause an instantaneous increase in AMP in the working muscles (Wegener et al., 1991). In concert with high levels of $\mathrm{F} 2,6 \mathrm{P}_{2}$, this rapid increase in AMP will then ensure high glycolytic rates, as required for take-off (Candy et al., 1997).

In contrast, inhibition of DUM neurons during flight (Duch and Pflüger, 1999) will allow octopamine levels in flight muscles to decrease, because this is the only octopaminergic innervation that they receive. In fact, such a decrease in octopamine in locust flight muscles does occur within the first $10 \mathrm{~min}$ of a flight (Goosey and Candy, 1982), despite increased hemolymph octopamine levels (Goosey and Candy, 1980). Decreased octopamine levels in flight muscle would be expected to also decrease F2,6 $\mathrm{P}_{2}$ levels in muscle, which in fact have been shown to decrease by $80 \%$ within the first $10 \mathrm{~min}$ of a flight (Wegener et al., 1986). With the other effectors of PFK unchanged, as demonstrated by NMR spectroscopy (Wegener et al., 1991), this will attenuate glycolytic flux, and correspondingly favor oxidizing lipids during prolonged flight. DUM neuron activity during flight would be expected to counteract the decrease in $\mathrm{F} 2,6 \mathrm{P}_{2}$. In fact, this hypothesis is supported by reports that the decrease in $\mathrm{F} 2,6 \mathrm{P}_{2}$ in working flight muscles can be reverted by injecting octopamine in flying locusts (Blau and Wegener, 1994) or incubating electrically stimulated flight muscles with octopamine (Blau et al., 1994). Therefore, we conclude that DUM neuron inhibition during flight (Duch and Pflüger, 1999) may be part of the mechanism to reduce glycolytic flux to favor the oxidation of fat during prolonged flight.

Central modulatory neurons hence appear to contribute to adjusting flight muscle metabolism to the preferential oxidation of carbohydrates (muscle glycogen and hemolymph trehalose) for take-off. Such a direct control of muscle metabolism by modulatory neurons has not been demonstrated before. This underlines the increasing awareness that the appropriate control of behavior requires numerous interactions between the nervous system and other organs as well as the environment. Neuromodulators, such as octopamine, are also released within the CNS, and central neurons are equipped with the appropriate sets of receptors. As Robertson and Steele (1973) have observed octopamine to mobilize glycogen in isolated cockroach ganglia, this prompts the question as to whether neuromodulators also support neurons to meet changing catabolic demands.

\section{References}

Albrecht FO (1953) The anatomy of the migratory locust. London: Athlone.

Blau C, Wegener G (1994) Metabolic integration in locust flight: the effect of octopamine on the glycolytic activator fructose 2,6-bisphosphate in flight muscle in vivo. J Comp Physiol [B] 164:11-15.

Blau C, Wegener G, Candy DJ (1994) The effect of octopamine on the glycolytic activator fructose 2,6-bisphosphate in perfused locust flight muscle. Insect Biochem Mol Biol 24:677-683.

Candy DJ, Becker A, Wegener G (1997) Coordination and integration of metabolism in insect flight. Comp Biochem Physiol [B] 117:497-512.

Duch C, Pflüger HJ (1999) DUM neurons in locust flight: a model system for amine-mediated peripheral adjustments to a central motor program. J Comp Physiol [A] 184:489-499.

Evans PD, Robb S (1993) Octopamine receptor subtypes and their modes of action. Neurochem Res 18:869-874.

Goosey MW, Candy DJ (1980) The D-octopamine content of the hemolymph of the locust, Schistocerca americana gregaria, and its elevation during flight. Insect Biochem 10:393-397.

Goosey MW, Candy DJ (1982) The release and removal of octopamine by tissues of the locust Schistocerca americana gregaria. Insect Biochem 12:681-685. 
Hildebrandt H, Müller U (1995) Octopamine mediates rapid stimulation of protein kinase A in the antennal lobe of honeybees. J Neurobiol 27:44-50.

Jutsum AR, Goldsworthy GJ (1976) Fuels for flight in Locusta. J Insect Physiol 22:243-249.

Krall S, Peveling R, Ba-Diallo D (1997) New strategies in locust control. Basel: Birkhäuser.

Krause U, Wegener G (1996) Control of glycolysis in vertebrate skeletal muscle during exercise. Am J Physiol 270:R821-R829.

Kutsch W, Schneider H (1987) Histological characterization of neurones innervating functionally different muscles of Locusta. J Comp Neurol 261:515-528.

Orchard I, Ramirez JM, Lange AB (1993) A multifunctional role for octopamine in locust flight. Annu Rev Entomol 38:227-249.

Rheuben MB (1995) Specific associations of neurosecretory or neuromodulatory axons with insect skeletal muscle. Am Zool 35:566-577.

Robb S, Cheek TR, Hannan FL, Hall LM, Midgley JM, Evans PD (1994) Agonist-specific coupling of a cloned Drosophila octopamine/tyramine receptor to multiple second messenger systems. EMBO J 13:1325-1330.

Robertson HA, Steele JE (1973) Effect of monophenolic amines on glycogen metabolism in the nerve cord of the American cockroach, Periplaneta americana. Insect Biochem 3:53-59.

Van der Horst DJ (1990) Lipid transport functions of lipoproteins in flying insects. Biochim Biophys Acta 1047:195-211.
Van der Horst DJ, Van Marrewijk WJA, Diederen HB (2001) Adipokinetic hormones of insects: release, signal transduction, and responses. Int Rev Cytol 211:179-240.

Van Schaftingen E (1984) D-Fructose 2,6-bisphosphate. In: Methods of enzymatic analysis, Vol 6: Metabolites 1: carbohydrates (Bergmeyer HU, Bergmeyer J, Grassl M, eds), pp 335-341. Weinheim, Germany: Wiley.

Van Schaftingen E, Lederer B, Bartrons R, Hers H-G (1982) A kinetic study of pyrophosphate: fructose 6-phosphate phosphotransferase from potato tubers. Eur J Biochem 129:191-195.

Wegener G (1996) Flying insects: model systems in exercise physiology. Experientia 52:404-412.

Wegener G, Krause U (2002) Different modes of activating phosphofructokinase, a key regulatory enzyme of glycolysis, in working vertebrate muscle. Biochem Soc Trans 30:264-270.

Wegener G, Michel R, Newsholme EA (1986) Fructose 2,6-bisphosphate as a signal for changing from sugar to lipid oxidation during flight in locusts. FEBS Lett 201:129-132.

Wegener G, Beinhauer I, Klee A, Newsholme EA (1987) Properties of locust muscle 6-phosphofructokinase and their importance in the regulation of glycolytic flux during prolonged flight. J Comp Physiol [B] 157:315-326.

Wegener G, Bolas NM, Thomas AAG (1991) Locust flight metabolism studied in vivo by ${ }^{31} \mathrm{P}$ NMR spectroscopy. J Comp Physiol [B] 161:247-256. 